\title{
Mechanical stimulation regulates differentiation of bone marrow stem cells by modulating miR-140-5p via TGF $\beta 1 /$ Smad2 signaling pathway
}

\section{Yuepeng Qian}

Third Affiiated Hospital of Southern Medical University

Guozheng Zhu

Southern Medical University Nanfang Hospital

\section{Zelin Ye}

Southern Medical University Nanfang Hospital

\section{Canjun Zeng}

Southern Medical University

\section{Runguang Li ( $\square$ lirunguang79@163.com )}

Department of Orthopedics, Third Affiliated Hospital of Southern Medical University, Academy of Orthopedics Guangdong Province https://orcid.org/0000-0002-4703-7180

\section{Research article}

Keywords: Osteoporosis, bone marrow mesenchymal stem cells (BMSCs), mechanical stimuli, miR-140$5 p$, TGFB1/Smad2

Posted Date: February 13th, 2020

DOI: https://doi.org/10.21203/rs.2.23417/v1

License: @ (i) This work is licensed under a Creative Commons Attribution 4.0 International License. Read Full License 


\section{Abstract}

Background: Osteoporosis is a common progressive bone disease that drastically impairs patient health, independent mobility, and quality of life, and there is an urgent need for improved preventive and therapeutic strategies. A shift in bone marrow mesenchymal stem cell (BMSC) differentiation from osteogenic to adipogenic may contribute to disease pathogenesis. Mechanical stress on BMSCs is reported to promote osteogenesis, so we examined the effects of mechanical stimulation on BMSC differentiation and associated signaling pathways.

Methods: A sinusoidal tensile stress loading device was developed and examined effects of mechanical (stretch) stimulation on cultured BMSC (isolated from Sprague-Dawley rats) phenotype under osteogenic and adipogenic culture conditions. Osteogenic differentiation of BMSCs was assessed by alkaline phosphatase (ALP) staining and expression of Runx2 and BMP2, while adipogenic differentiation was evaluated by oil red $O$ staining and expression of PPARY and C/EBPa.

Results: It demonstrated that appropriate mechanical stimulation could promote osteogenic differentiation of BMSCs and inhibit differentiation into adipocytes. The mechanic stimuli could inhibit the expression of miR-140-5P in BMSCs, and the overexpression of miR-140-5P inhibited the osteogenic differentiation, whereas the inhibition of miR-140-5P promoted the osteogenic differentiation. Further, both mechanical stimulation and miR-140-5p knockdown promoted TGF $\beta 1 /$ Smad2 signaling, while miR140-5p overexpression downregulated TGF $\beta 1 /$ Smad2 signaling.

Conclusions: Appropriate mechanical stimulation promoted osteogenic differentiation and inhibited the adipogenic differentiation of BMSCs by lowering miR-140-5p expression, which in turn upregulates the TGF $\beta 1 /$ Smad2 signaling pathway. Our results provide a foundation for the development of effective strategies to promote bone remodeling, thereby lowering the burden of osteoporosis.

\section{Background}

Osteoporosis is a common progressive disease characterized by bone fragility due to the degradation of bone microstructure and decreased bone mass [1], which severely impacts health, mobility, and general quality of life [2]. Although the precise pathogenesis remains unclear, the fundamental mechanism underlying osteoporosis is an imbalance between bone formation and resorption [3]. Osteoblasts derived from bone marrow mesenchymal stem cells (BMSCs) serve to replenish bone tissue. However, BMSCs can differentiate into a variety of cell types aside from osteoblasts, including chondrocytes, myocytes, and adipocytes $[4,5]$. In general, the shift in the differentiation from the osteogenic to adipogenic lineage increases with age, which may impair bone remodeling and reduce osteoblast formation, leading to the development of osteoporosis [6, 7].

Accordingly, most current drug treatments for osteoporosis inhibit bone resorption, accelerate bone formation, or promote bone mineralization. However, long-term use of these drugs has certain limitations. For instance, hormone replacement therapy may increase the risk of cardiovascular diseases [8], while 
bisphosphonate therapy may cause severe bone turnover suppression and mandible osteonecrosis [9]. Thus, there is a critical need for improved preventive and therapeutic strategies for osteoporosis.

Most tissues are subjected to mechanical stimuli that regulate biological functions such as proliferation and differentiation [10]. Previous studies have shown that lack of exercise and the associated reduction in stem cell mechanical stimulation decreases osteogenic differentiation and increases the risk of osteoporosis [11, 12]. Indeed, our previous studies have demonstrated that mechanical stress can promote osteogenic differentiation and inhibit adipogenic differentiation of BMSCs $[13,14]$. Further understanding of how mechanical stimuli, especially mechanical stress, affect skeletal tissue differentiation will provide insight into bone repair processes that may be exploited for novel therapeutic strategies. Several studies examining the relationships between mechanical stimulation and molecular expression profiles during bone healing have reported that non-coding microRNAs (miRNAs) participate in the regulation of skeletal growth and development. For instance, miR-140-5p has been reported to regulate cartilage development and bone homeostasis as well as contribute to age-related joint disease [15]. MicroRNA-140-5p was also found to be upregulated in murine primary osteoblasts and a vitamin Dtreated osteoblast cell line [16]. A very recent study provided evidence that miR-140-5p regulates temporomandibular joint osteoarthritis (TMJ-OA) pathogenesis through the TGF- $\beta / S m a d 2$ signaling pathway [17]. Furthermore, miR-140-5p was reported to promote osteogenesis of ACSs by directly regulating toll-like receptor 4 (TLR4) and bone morphogenic protein 2 (BMP2), resulting in enhanced fracture healing and bone formation in the atrophic nonunion rat model [18]. However, is still unknown whether miR-140-5p influences osteogenic or adipogenic differentiation of BMSCs in response to mechanical stimulation. The purpose of this study is to investigate the effects of mechanical stimulation on osteogenic and adipogenic differentiation of BMSCs and to explore the underlying molecular mechanisms, including the contributions of miR-140-5p and TGF- $\beta / S m a d 2$ signaling.

\section{Methods}

\section{Isolation and culture of rat BMSCs}

Bone marrow stem cells were isolated from Sprague-Dawley rats (Experimental animal center of Nanfang Hospital $\llbracket$ male or female, $80-100 \mathrm{~g}$. All rats were killed by cervical dissection, and the bodies were collected and burned by environmental health management department of Guangdong province.) by flushing the femurs and tibias with DMEM-LG medium (Gibco, Langley, OK, USA) supplemented with 10\% defined fetal calf serum (Gibco), $100 \mathrm{U} / \mathrm{ml}$ penicillin, and $100 \mu \mathrm{g} / \mathrm{ml}$ streptomycin (North China Pharmaceutical Factory, China) (termed general medium, GM). Isolated cells were plated in the some medium on $25 \mathrm{~cm}^{2}$ flasks and incubated at $37{ }^{\circ} \mathrm{C}$ under a humidified atmosphere containing $5 \% \mathrm{CO}_{2}$. After $24 \mathrm{~h}$, non-adherent cells were removed by washing with PBS, and fresh GM was added to allow for further growth. The culture medium was changed every 2-3 days thereafter. When the cells reached $80 \%-90 \%$ confluence, they were washed with PBS, detached by $0.25 \%$ trypsin, and subcultured in new 25 $\mathrm{cm}^{2}$ flasks at $1 \times 10^{4}$ cells $/ \mathrm{cm}^{2}$. Cells were collected at the second or third generation and sent for flow 
cytometry analysis (BD, Franklin Lakes, NJ, USA) of CD29, CD34, CD44, and CD45 expression for confirmation of BMSC phenotype $[13,14]$.

\section{Induction of osteogenesis or adipogenic differentiation and delivery of mechanical stimuli}

Bone marrow stem cells at the logarithmic growth phase were collected, washed, and resuspended in DMEM at $1 \times 10^{5}$ cells $/ \mathrm{ml}$. Cells were seeded onto Bioflex 6 -well plates at $1.5-2 \mathrm{ml}$ per well and incubated under $5 \% \mathrm{CO}_{2}$ at $37^{\circ} \mathrm{C}$. Control cultures were refreshed with basal medium every $48 \mathrm{~h}$. Differentiation of BMSCs was induced using the culture conditions previously described [19]. For osteogenic induction, cells were cultured in medium supplemented with $10 \% \mathrm{FBS}, 1 \%$ penicillin/streptomycin, $100 \mathrm{nmol} / \mathrm{L}$ dexamethasone, $50 \mu \mathrm{mol} / \mathrm{L}$ ascorbic acid 2-phosphate, and $10 \mathrm{mmol} / \mathrm{L} \beta$-glycerophosphate. For adipogenic induction, cells were cultured in DMEM containing 10\% FBS, $1 \%$ penicillin/streptomycin, 1 $\mu \mathrm{mol} / \mathrm{L}$ dexamethasone, $0.5 \mathrm{mmol} / \mathrm{L}$ IBMX, $10 \mathrm{mg} / \mathrm{L}$ insulin, and $200 \mu \mathrm{mol} / \mathrm{L}$ indomethacin. Mechanical stimulation parameters were set to $5 \%$ deformation rate, $0.5 \mathrm{~Hz}$, and $6 \mathrm{~h} / \mathrm{d}$. The mechanical stretching device was updated to stimulate cells as previously described [20].

\section{Alkaline phosphatase and oil red 0 staining}

An ALP staining kit (Tiangen Biotech Co. Ltd., Beijing, China) was used for determination of osteogenic differentiation according to the manufacturer's instructions. Briefly, cells were fixed with $4 \%$ paraformaldehyde in PBS for $12 \mathrm{~min}$ at room temperature then stained with 1-2 ml/well ALP solution for $30 \mathrm{~min}$ at room temperature. The solution was aspirated and the cells were washed with distilled water and observed under light microscopy for ALP-positive cells. For determination of adipogenic differentiation, cells were fixed with $10 \%$ neutral buffered formalin for $1 \mathrm{~h}$ at room temperature, incubated with $60 \%$ isopropanol for $1 \mathrm{~min}$, and then stained with oil red 0 for $15 \mathrm{~min}$. Positively stained lipid droplets (red) were visualized under light microscopy.

\section{Western Blotting}

Cells were lysed by incubation in RIPA buffer containing protease inhibitor cocktail (Roche, Shanghai, China) on ice for $30 \mathrm{~min}$. Cell lysates were centrifuged at $12000 \mathrm{~g}$ for $5 \mathrm{~min}$ at $4^{\circ} \mathrm{C}$, and the supernatant was collected. Protein concentration was determined using the Pierce BCA protein Assay Kit (Thermo Scientific, MD, USA). Total protein was separated by SDS-PAGE ( $25 \mu \mathrm{g}$ per gel lane) and transferred onto polyvinylidene fluoride (PVDF) membranes (Millipore, MA, USA). Membranes were blocked with $5 \%$ nonfat milk in TBST buffer and probed with primary antibodies against the osteogenic markers Runx2 (Biorbyt, CA, USA) and BMP2 (ab14933, Abcam) or the bone adipogenic markers PPARY (ab45036, Abcam) and C/EBPa (ab40764, Abcam). In addition, membranes were incubated with anti-Smad2 (ab33875, Abcam), anti-TGFB1 (ab179695, Abcam), and anti-TGFR1 (ab31013, Abcam) to assess expression of TGF $\beta 1 /$ Smad2 signaling pathway components. Subsequently, membranes were washed with TBST, and incubated with horseradish peroxidase (HRP)-conjugated secondary antibodies (115-035003 and 111-035-003 from Jackson Immuno Research Laboratories, West Grove, PA, USA). Target 
proteins were visualized using a ECL chemiluminescence kit (Pierce) and band densities quantified using ImageJ (National Institutes of Health, USA).

\section{RNA Extraction and RT-PCR Analysis}

The expression levels of osteoblastic and adipocyte genes were analyzed by quantitative real-time PCR. Briefly, BMSCs were harvested and RNA was extracted using Trizol Reagent. Total RNA was reverse transcribed to cDNA using kit K1622 (Thermo Scientific). Real-time PCR assays were performed using Allin-One $^{\mathrm{TM}}$ qPCR reagents (Genecopoeia, Guangzhou, China) with specific primers. The primer sequences for the differentiation markers were as follows: Runx2-F, GGACCGACACAGCCATATAAA, Runx2-R, GCCTCATTCCCTAACCTGAAA; BMP2-F, CAGTGGGAGAGCTTTGATGT, BMP2-R, ACCTGGCTTCTCCTCTAAGT; PPARY-F, GACCTGAAGCTCCAAGAATACC, PPARY-R, TTCATGTGGCCTGTTGTAGAG; C/EBP-F, CCTCTGGGATGGATCGATTATG, C/EBP-R, GGGACCTTAGTTTCTGGTCTTG. The primer sequences for the TGF $\beta 1 /$ Smad2 signaling pathway were as follows: Smad2-F, GAGCACGTGAGGTGAGATTT, Smad2-R, CTAAGGACTTCCAGAGGGAAAC; TGFß1-F, GCAACAATTCCTGGCGTTAC, TGFß1-R, GTATTCCGTCTCCTTGGTTCAG. TGFßr1-F, CTTCCTTGAGTCACTGGGTATC, and TGFßr1-R, CTTGGCTGTCACCCTAATCTT. The expression levels of target genes were normalized to expression of the GAPDH gene.

\section{MiR-140-5p transient overexpression and inhibition}

To assess the effects of miR-140-5p on differentiation, cells were transiently transfected with a vector encoding miR-140-5p mimic or inhibitor (GenePharma, Suzhou, China). Briefly, BMSCs were expanded in culture until $80 \%$ confluent, trypsinized, reseeded in 6-well Bioflex plates, washed with PBS, and treated with transfection medium containing riboFECT CP Reagent (RiboBio, Guangzhou, China) and $100 \mathrm{~nm}$ of either miR-140-5p mimic, inhibitor, or scrambled miR-NC (control) for approximately $48 \mathrm{~h}$. Total RNA was then extracted for RT-PCR analysis and proteins were extracted for western blot analysis as described.

\section{Luciferase assays of miR-140-5p expression}

The putative target sites for miR-140-5p on the 3'-UTR of TGF $\beta R 1$ were predicted using bioinformatics tools. HEK293 cells were seeded on 24 -well plates and cultured until $60 \%$ confluent. The cells were then transfected with a vector encoding wild type or mutated TGFBR1 3'-UTR using the X-treme GENE ${ }^{\text {TM }} \mathrm{HP}$ DNA Transfection Reagent. After incubation for $48 \mathrm{~h}$, the cells were lysed in 1× Passive Lysis Buffer and luciferase activities measured using the Dual-Luciferase ${ }^{\circledR}$ Reporter Assay System (Promega, Madison, WI, USA) according to the manufacturer's instructions.

\section{Statistical analysis}

All data shown are expressed as mean \pm standard deviation (SD) of at least three independent experiments. Group means were compared by independent sample t-tests using Prism version 6 (GraphPad software). A $p \leq 0.05$ (two-tailed) was considered statistically significant for all tests. 


\section{Results}

\section{Effects of mechanical stimulation on BMSC differentiation}

Bone marrow stem cells were induced towards osteogenic or adipogenic differentiation using specific culture conditions with or without additional mechanical stimulation (stretch stress). After 5 days in culture, RT-qPCR and western blotting (WB) were performed to measure expression levels of the BMSC osteogenic markers Runx2 and BMP2 and the adipogenic markers PPARY and CEBPa. As demonstrated in Figure 1A, cells cultured under osteogenic conditions with mechanical stimulation (stretch stress) exhibited 1.22-fold higher Runx2 mRNA expression and 1.27-fold higher BMP2 mRNA expression than the corresponding no stress group as measured by RT-qPCR (Figure 1Aa-b). Consistent with gene expression levels, WB revealed greater Runx2 and BMP2 protein expression levels in the stretch stress group compared to the no stress group (Figure 1Ac-d). Moreover, cells cultured under adipogenic conditions with stretch stress showed significantly reduced expression levels of the adipogenic marker genes PPARY and CEBPa than the corresponding no stress group ( $70 \%$ and $72 \%$, respectively, of no stress group values, Figure 1Ba-b), while WB demonstrated parallel reductions in protein expression (Figure 1Bc-d). These results suggest that appropriate stretch stress can promote osteogenic differentiation and inhibit adipogenic differentiation of BMSCs.

\section{Expression of miR-140-5p during osteogenic and adipogenic differentiation of BMSCs}

To explore the possible molecular mechanisms underlying the effects of mechanical stimulation on BMSC differentiation, we first assessed the expression levels of miR-140-5p. As shown in Figure $2 a$ and b, miR-140-5p expression gradually decreased and reached a nadir on the third day of osteogenic differentiation. Alternatively, miR-140-5p expression increased significantly during adipogenic differentiation, reaching a peak on the third day, followed by a slight decrease. On day 3 , the expression level of miR-140-5p was $20 \%$ of control in the osteogenic condition and 5.6-fold higher than control in the adipogenic condition. Thus, miR-140-5p expression app-ears to suppress osteogenic and (or) promote adipogenic differentiation.

We therefore examined if mechanical stress influences miR-140-5p expression. As shown in Figure $2 c$ and d, BMSCs cultured under osteogenic conditions plus stretch stress (A1) demonstrated $24 \%$ lower miR-140-5p expression than BMSCs under osteogenic culture without stretch stress (B1), and miR-140-5p expression levels in both osteogenic BMSC groups (A1 and B1) were significantly lower than in BMSCs without induction (A3 and B3). In BMSCs cultured under adipogenic conditions, stretch stress (A2) reduced miR-140-5p expression (by 39\%) compared to adipogenic BMSCs without stress (B2). In addition, stretch stress reduced miR-140-5p expression under non-induction culture conditions.

Collectively, these results indicate that the expression level of miR-140-5P is downregulated during osteogenic differentiation of BMSCs and further inhibited by appropriate stretch stress.

\section{Effects of miR-140-5p overexpression on osteogenic and adipogenic differentiation of BMSCs}


To provide further evidence for miR-140-5p function in BMSC osteogenic and adipogenic differentiation, we examined the effects of culture condition and stretch stress in BMSCs transfected with a miR-140-5p mimic. RT-PCR on day 3 of induction confirmed miR-140-5p overexpression (Figure 3Aa). Moreover, expression levels of the osteogenic marker genes Runx2 and BMP2 were significantly lower in BMSCs overexpressing miR-140-5p than osteogenic BMSCs transfected with control vector (Figure 3Ac-d), suggesting that miR-140-5p negatively regulates osteogenic differentiation of BMSCs. Further, under adipogenic differentiation conditions, expression levels of the adipogenic marker genes PPAR $\square$ and C/EBPa were significantly upregulated in BMSCs overexpressing miR-140-5p (Figure 3Ba-b). After 7 days of differentiation under osteogenic conditions, BMSCs overexpressing miR-140-5p (A1 group) also exhibited lower ALP staining intensity than osteogenic BMSCs expressing control vector (A2 group) (Fig. 3C). Conversely, under adipogenic culture conditions, BMSCs overexpressing miR-140-5p (C1 group) demonstrated more intense oil red $\mathrm{O}$ staining than adipogenic BMSCs expressing control vector (C2 group). These results suggest that miR-140-5p promotes adipogenic differentiation of BMSCs rather than osteogenic differentiation.

\section{Effects of miR-140-5p knockdown on osteogenic and adipogenic differentiation of BMSCs}

We then examined the effect of miR-140-5p knockdown on BMSC differentiation by transfection of a miR140-5p inhibitor. RT-PCR confirmed knockdown of miR-140-5p compared to control vector (Figure 4Aa). Under osteogenic culture conditions, BMSCs expressing the miR-140-5p inhibitor exhibited significantly higher expression levels of Runx2 and BMP2 than BMSCs expressing control vector (Figure 4Ac-d), while under adipogenic conditions, BMSCs expressing the miR-140-5p inhibitor demonstrated significantly downregulated PPARY and C/EBPa expression compared to controls (Figure 4Ba-b). Under osteogenic conditions, BMSCs expressing the miR-140-5p inhibitor (A1 group) also exhibited greater ALP staining than cells expressing control vector (A2 group), while under adipogenic conditions, BMSCs with miR-140$5 p$ knockdown (C1 group) exhibited lighter oil red O staining than BMSCs expressing control vector (C2 group). These results provide further evidence that miR-140-5p suppresses osteogenic differentiation of BMSCs and promotes adipogenic differentiation

\section{The participation of TGF $\beta 1 /$ Smad2 signaling pathway}

Furthermore, we try to find out the pathway signal targeted to the mechanism that miR-140-5p regulate to BMSCs differentiation. With the help of biomedical date source website, we decided to choose TGF$1 /$ Smad2 as our target signal pathway to perform further research. Firstly, luciferase reporter assay was performed to detect the direct binding between miR-140-5P and TGF $\beta r 1$. We found that ectopic expression of miR-140-5P significantly decreased the luciferase signal of 3' UTR of WT TGF $\beta r 1$ compared to the miR-NC. This suppressive effect was abolished by mutated miR-194 binding site of 3' UTR TGF $\beta r 1$. Secondly, The WB expression of TGF-1/Smad2 signal transduction pathway markers significantly downregulate in the BMSCs with adipogenic as significantly upregulated with osteogenic (Figure 5).

These results indicated the participating role of TGF $31 /$ Smad2 signaling pathway in the regulatory role of miR-140-5P on the BMSCs differentiation. 


\section{The expression level of TGF-1/Smad2 signal transduction pathway markers in BMSCs change as miR- $140-5 p$ overexpression and inhibition under mechanical stimulation}

To explore the connection between miR-140-5P and TGF-1/Smad2 signal pathway, we transfected the miR-140-5P mimic and inhibitor into BMSCs separately and found TGF-1/Smad2 pathway were influenced by the change activity of miR-140-5P. As mentioned above, during the osteogenic differentiation of BMSCs, the activity of TGF $31 / \mathrm{Smad} 2$ marks was significantly promoted under stress. As shown in Figure $6 \mathrm{~A}$, this effect was further promoted when agonizing miR-140-5p, which was antagonized in inhibiting miR-140-5p. Conversely, it was observed in adipogenic differentiation that stress inhibited the activity of TGF $\beta 1 / \mathrm{Smad} 2$ channels, while the effect is antagonized by a 140-5p inhibitor (Figure 6B). This suggests that the regulation of the TGF-1/Smad2 pathway by miR-140-5p is involved in the regulation of stress on the differentiation of BMSCs.

\section{Discussion}

Osteoporosis is a systemic bone disease characterized by reduced bone mineral density and bone mass [21]. During the development of osteoporosis, the proportion of BMSCs differentiated into solitary cells is reduced and bone marrow is gradually replaced by adipose tissue [22]. Previous studies have shown that adipocytes and osteoblasts share a common progenitor cell [23]. Under the appropriate mechanical (stretch) stimulation, a variety of extracellular matrix components are secreted in the stretch zone and BMSCs differentiate into osteoblasts [24]. Consistent with previous research results, we demonstrate that appropriate mechanical stimulation can promote osteogenic differentiation of BMSCs and inhibit the adipogenic differentiation of BMSCs[13, 14]. Adipogenic differentiation was associated with enhanced expression of miR-140-5p and concomitant downregulation of TGF $\beta 1 /$ Smad2 signaling components (TGF $\beta 1, T G F \beta R 1$, and Smad2), while both mechanical stimulation and osteogenic culture suppressed miR-140-5p expression and upregulated expression of TGF $\beta 1 / \mathrm{Smad} 2$ signaling components.

Stress exists in all intracellular environments, regulating cell biological functions, such as cell proliferation and differentiation, and appropriate stress stimulation is particularly important in bone regeneration after fracture[25]. To further study the effects of mechanical stimulation on the differentiation of bone marrow stem cells in vitro, we developed a sinusoidal tensile stress loading machine suitable for stretch stimulation of cultured BMSCs[20]. Our results clearly show that appropriate mechanical stimulation can promote osteogenic differentiation of BMSCs as indicated by deeper ALP staining and upregulation of osteogenic markers Runx2 and BMP2. Runx2 is a member of the Runxx family of transcription factors and acts as a specific transcription factor for bone cells during development and remodeling [26], while BMP-2 is a member of the TGF $\beta$ structure-related protein superfamily that can induce bone and cartilage formation in combination with bone conduction carriers such as collagen and synthetic hydroxyapatite [27]. Our results also demonstrated that the same extent of mechanic stimuli inhibited the adipogenic differentiation of BMSCs, indicated by the lower red oil 0 staining, as well as upregulated expressions of osteogenic gene markers including PPAR- $y$ and C/EBPa, which are the most important transcription factors in MSCs during adipogenic differentiation. Our results 
were consistent with previous study by Turner et al., which showed that the appearance of intracellular lipid droplets was delayed in the stress group during the differentiation and maturation of MSCs into adipocytes [28], and supported the statement that shear stresses at physiological levels can differentiate MSCs into endothelial cells by inducing the expression of endothelial-cell-specific markers [29].

The early phase of BMSC differentiation involves changes in signaling pathways that in turn alter responses to external factors. MicroRNAs are small non-coding RNA of about 20 to $24 \mathrm{nt}$ [30] with diverse functions in stem cells including regulation of development, differentiation, proliferation, and apoptosis. MicroRNA-503-5p has been shown to inhibit osteogenic differentiation of stem cells under stress [31]. Wang et al. used gene chips to detect changes in miRNAs during stem cell differentiation, and found that the expression level of miR-140 increased significantly during adipogenic differentiation [32]. Similarly, Zhang and colleagues demonstrated that the expression level of miR-140-5p was three times higher during the third stage of adipogenic differentiation of adipose-derived stem cells compared to control cells [33], suggesting the varied regulatory roles of the same miRNA in the differentiation of stem cells. To elucidate the underlying mechanisms, we examined the expression of miR-140-5p at different stages of BMSC differentiation, the effects of miR-140-5p overexpression and knockdown on differentiation, and the associations among miR-14-5p expression, osteogenic and adipogenic marker expression, and TGF $\beta 1 /$ Smad2 signaling component expression under osteogenic and adipogenic culture conditions in the presence and absence of mechanical stress. Under osteogenic culture conditions, mechanical stimulation inhibited the expression of miRNA-140-5p, upregulated TGF $\beta 1 /$ Smad2 signaling components, and promoted osteogenic differentiation of BMSCs, proving a possible explanation for the benefits of appropriate mechanical stress on bone remodeling.

In accord with our results, Liu at al. reported that reduced expression of miR-503-5p promoted the osteogenic differentiation of bone marrow stromal cells under stress [31]. Other miRNAs that regulate stem cell differentiation and are influenced by mechanical stress include miR-34 s, miR-29b, and miR-30a [34-36]. Various miRNAs can have unique regulatory effects on osteogenic differentiation and respond differently to mechanical stress. Overexpression and knockdown of miR-140-5p in BMSCs resulted, respectively, in enhanced osteogenic and adipogenic differentiation. In addition, TGF $\beta$ R1 expression was reduced by miR-140-5p overexpression compared to negative control cells under both culture conditions in the presence and absence of mechanical stress; in contrast, TGF $\beta$ R 1 expression was enhanced by miR140-5p knockdown under all conditions. Furthermore, luciferase reporter assay confirmed direct binding between miR-140-5p and the TGF $\beta R 1$ promoter. Intriguingly, TGF $\beta$ R1 expression was elevated by mechanical stress compared to the corresponding unstressed group regardless of miR-140-5p expression level, indicating that both stress and miR-140-5p can regulate BMSC differentiation through reciprocal regulation of the TGF $\beta 1 /$ Smad2 signaling pathway.

Taken together, mechanical stimuli promote osteogenic differentiation of BMSCs, in combing with lower expression of miR-140-5p via TGF $\beta 1 /$ Smad2 signaling pathway, suggesting the positive role of mechanical stimuli in the differentiation of BMSCs towards osteoblasts. 


\section{Conclusions}

Appropriate mechanical stimulation promotes the osteogenic differentiation of BMSCs by lowering the expression of miR-140-5P, which in turn upregulates the TGF $31 / \mathrm{Smad} 2$ signaling pathway and downstream osteogenic genes. Our results provide a plausible explanation for the beneficial effects of mechanical stimulation on bone remodeling and a foundation for the development of more effective strategies to treat osteoporosis.

\section{Abbreviations}

BMSCs: bone marrow mesenchymal stem cells

ALP: alkaline phosphatase

BMP2: bone morphogenetic protein-2

C/EBPa: Recombinant Human CCAAT/enhancer binding protein

PPARY: peroxisome proliferators-activated receptor-y

RUNX2: Runt-related transcription factor 2

Smad2: drosophila mothers against decapentaplegic2

TGF- $\beta$ : transforming growth factor- $\beta$

\section{Declarations}

\section{Ethics statement}

Ethics approval and consent to participate: All animal experiments were conducted according to relevant national and international guidelines and approved by the Animal Care and Use Committee of Southern Medical University. Appropriate steps were taken to ameliorate suffering.

\section{Acknowledgement}

We sincerely appreciated all workers in this study

\section{Conflicts of interest:}

None

\section{Consent for publication:}

None 


\section{Authors' contributions:}

YP Q and GZ Z, ZL Y carried out the experiment. YP Q and GZ Z. wrote the manuscript with support from $R G L$ and CJ Z. RG $L$ and CJ Z. supervised the project. All authors have read and approved the manuscript.

\section{Funding}

National Natural Sciences Foundation of China (Approval no. 81101366), contribution to the design of the study and collection.

Natural Science Foundation of Guangdong Province (Approval no. 2018A030313640), contribution to the preparation of experimental materials.

Natural Science Foundation of Guangdong Province (Approval no. 2019A1515012176), contribution to the interpretation of data and in writing the manuscript.

\section{References}

1. Raisz LG. Pathogenesis of osteoporosis: concepts, conflicts, and prospects. J Clin Invest 2005; 115: 3318-25.

2. Xia WB, He SL, Xu L et al. Rapidly increasing rates of hip fracture in Beijing, China. J Bone Miner Res 2012; 27: 125-9.

3. Wang C, Meng H, Wang X et al. Differentiation of Bone Marrow Mesenchymal Stem Cells in Osteoblasts and Adipocytes and its Role in Treatment of Osteoporosis. Med Sci Monit 2016; 22: 22633.

4. Ankrum JA, Ong JF, Karp JM. Mesenchymal stem cells: immune evasive, not immune privileged. Nat Biotechnol 2014; 32: 252-60.

5. Mahla RS. Stem Cells Applications in Regenerative Medicine and Disease Therapeutics. Int J Cell Biol 2016; 2016: 6940283.

6. Bethel M, Chitteti BR, Srour EF, Kacena MA. The changing balance between osteoblastogenesis and adipogenesis in aging and its impact on hematopoiesis. Curr Osteoporos Rep 2013; 11: 99-106.

7. Kim M, Kim C, Choi YS et al. Age-related alterations in mesenchymal stem cells related to shift in differentiation from osteogenic to adipogenic potential: implication to age-associated bone diseases and defects. Mech Ageing Dev 2012; 133: 215-25.

8. Lewiecki EM. Treatment of osteoporosis with denosumab. Maturitas 2010; 66: 182-6.

9. Spanou A, Lyritis GP, Chronopoulos E, Tournis S. Management of bisphosphonate-related osteonecrosis of the jaw: a literature review. Oral Dis 2015; 21: 927-36.

10. Palomares KT, Gleason RE, Mason ZD et al. Mechanical stimulation alters tissue differentiation and molecular expression during bone healing. J Orthop Res 2009; 27: 1123-32. 
11. Dowthwaite GP, Flannery CR, Flannelly $\mathrm{J}$ et al. A mechanism underlying the movement requirement for synovial joint cavitation. Matrix Biol 2003; 22: 311-22.

12. Tagil M, Aspenberg P. Cartilage induction by controlled mechanical stimulation in vivo. J Orthop Res 1999; 17: 200-4.

13. Li R, Liang L, Dou Y et al. Mechanical strain regulates osteogenic and adipogenic differentiation of bone marrow mesenchymal stem cells. BioMed research international 2015; 2015: 873251.

14. Li R, Liang L, Dou Y et al. Mechanical stretch inhibits mesenchymal stem cell adipogenic differentiation through TGFbeta1/Smad2 signaling. J Biomech 2015; 48: 3665-71.

15. Miyaki S, Sato T, Inoue A et al. MicroRNA-140 plays dual roles in both cartilage development and homeostasis. Genes Dev 2010; 24: 1173-85.

16. Luo W, Liu L, Yang L et al. The vitamin D receptor regulates miR-140-5p and targets the MAPK pathway in bone development. Metabolism: clinical and experimental 2018; 85: 139-50.

17. Li W, Zhao S, Yang H et al. Potential Novel Prediction of TMJ-OA: MiR-140-5p Regulates Inflammation Through Smad/TGF-beta Signaling. Frontiers in pharmacology 2019; 10: 15.

18. Guo PY, Wu LF, Xiao ZY et al. Knockdown of MiR-140-5 promotes osteogenesis of adipose-derived mesenchymal stem cells by targeting TLR4 and BMP2 and promoting fracture healing in the atrophic nonunion rat model. European review for medical and pharmacological sciences 2019; 23: 2112-24.

19. Maridas DE, Rendina-Ruedy E, Le PT, Rosen CJ. Isolation, Culture, and Differentiation of Bone Marrow Stromal Cells and Osteoclast Progenitors from Mice. J Vis Exp 2018.

20. Zhu G, Qian Y, Wu W, Li R. Negative effects of high mechanical tensile strain stimulation on chondrocyte injury in vitro. Biochemical and biophysical research communications 2019; 510: 48-52.

21. Iseme RA, McEvoy M, Kelly B et al. Is osteoporosis an autoimmune mediated disorder? Bone Rep 2017; 7: 121-31.

22. Kawai M, de Paula FJ, Rosen CJ. New insights into osteoporosis: the bone-fat connection. J Intern Med 2012; 272: 317-29.

23. Liu ZJ, Zhuge Y, Velazquez OC. Trafficking and differentiation of mesenchymal stem cells. J Cell Biochem 2009; 106: 984-91.

24. Ai-Aql ZS, Alagl AS, Graves DT et al. Molecular mechanisms controlling bone formation during fracture healing and distraction osteogenesis. J Dent Res 2008; 87: 107-18.

25. Li R, Chen B, Wang $G$ et al. Effects of mechanical strain on oxygen free radical system in bone marrow mesenchymal stem cells from children. Injury 2011; 42: 753-7.

26. Bruderer M, Richards RG, Alini M, Stoddart MJ. Role and regulation of RUNX2 in osteogenesis. Eur Cell Mater 2014; 28: 269-86.

27. Schmitt B, Ringe J, Haupl T et al. BMP2 initiates chondrogenic lineage development of adult human mesenchymal stem cells in high-density culture. Differentiation 2003; 71: 567-77.

28. Turner NJ, Jones HS, Davies JE, Canfield AE. Cyclic stretch-induced TGFbeta1/Smad signaling inhibits adipogenesis in umbilical cord progenitor cells. Biochem Biophys Res Commun 2008; 377 : 
1147-51.

29. Dan P, Velot E, Decot V, Menu P. The role of mechanical stimuli in the vascular differentiation of mesenchymal stem cells. J Cell Sci 2015; 128: 2415-22.

30. Li N, Long B, Han W et al. microRNAs: important regulators of stem cells. Stem Cell Res Ther 2017; 8 : 110.

31. Liu L, Liu M, Li R et al. MicroRNA-503-5p inhibits stretch-induced osteogenic differentiation and bone formation. Cell Biol Int 2017; 41: 112-23.

32. Wang J, Guan X, Guo F et al. miR-30e reciprocally regulates the differentiation of adipocytes and osteoblasts by directly targeting low-density lipoprotein receptor-related protein 6. Cell Death Dis 2013; 4: e845.

33. Zhang $X$, Chang A, Li Y et al. miR-140-5p regulates adipocyte differentiation by targeting transforming growth factor-beta signaling. Sci Rep 2015; 5: 18118.

34. Papathanasiou I, Trachana V, Mourmoura E, Tsezou A. DNA methylation regulates miR-140-5p and miR-146a expression in osteoarthritis. Life sciences 2019; 228: 274-84.

35. Vasanthan P, Govindasamy V, Gnanasegaran N et al. Differential expression of basal microRNAs' patterns in human dental pulp stem cells. J Cell Mol Med 2015; 19: 566-80.

36. Wei J, Shi Y, Zheng L et al. miR-34s inhibit osteoblast proliferation and differentiation in the mouse by targeting SATB2. J Cell Biol 2012; 197: 509-21.

\section{Figures}




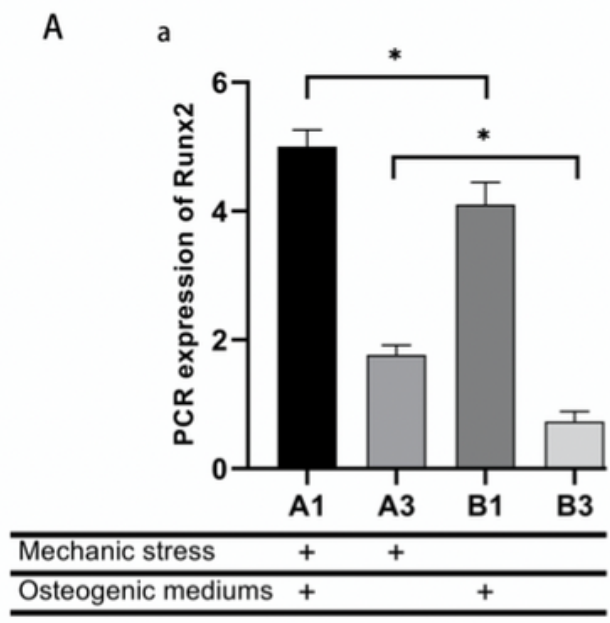

c

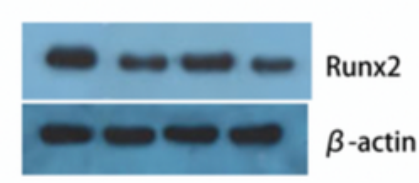

$\begin{array}{llll}\text { A1 } & \text { A3 } & \text { B1 } & \text { B3 }\end{array}$

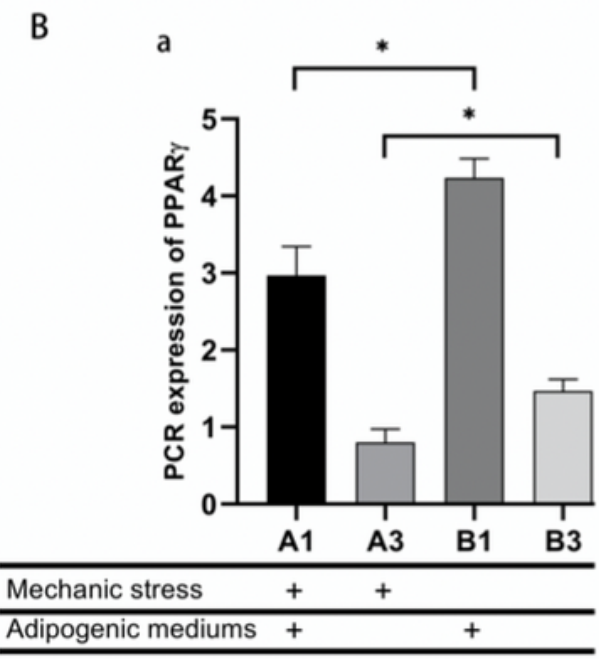

c

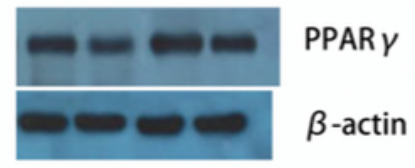

A1 A3 B1 B3

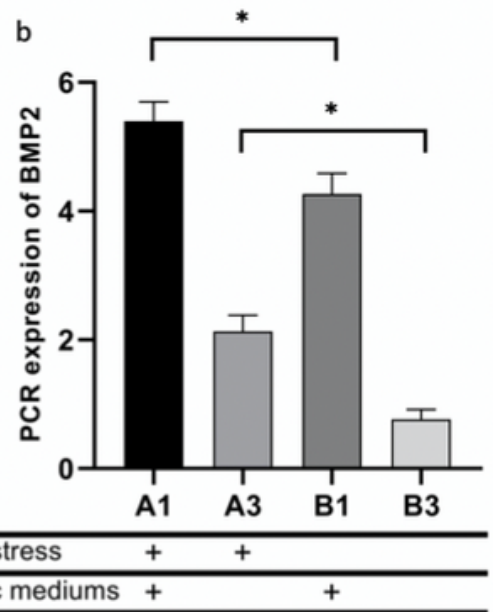

d

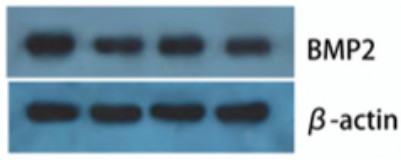

$\begin{array}{llll}\text { A1 } & \text { A3 } & \text { B1 } & \text { B3 }\end{array}$

b

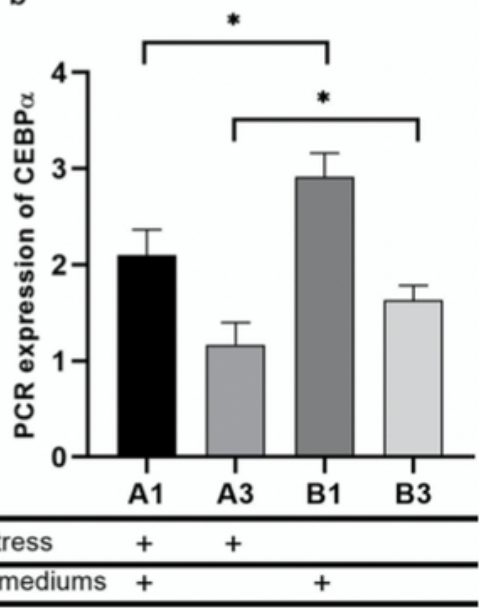

d

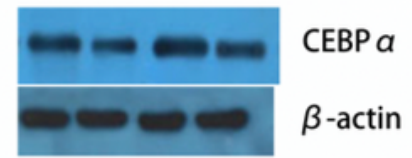

A1

\section{Figure 1}

Mechanical stimulation (stretch stress) promotes osteogenic differentiation and suppresses adipogenic differentiation of bone marrow stem cells (BMSCs). A) Expression levels of the bone osteoblast markers Runx2 and BMP2 under osteogenic culture conditions in the presence $(+)$ and absence of stretch stress. B) Expression levels of the bone adipogenic markers PPARY and CEBPa under adipogenic culture conditions in the presence and absence of stretch stress. 
a
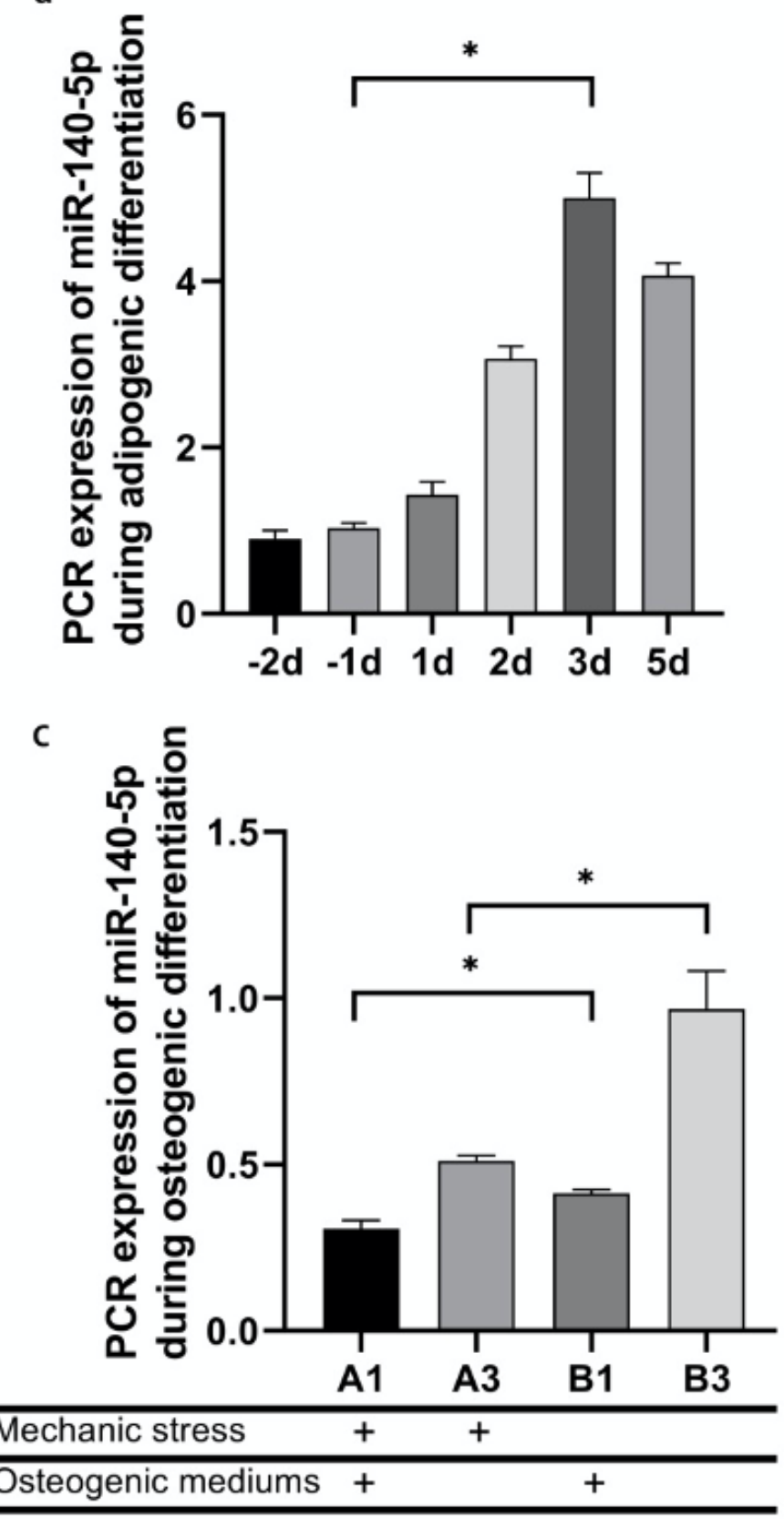

b

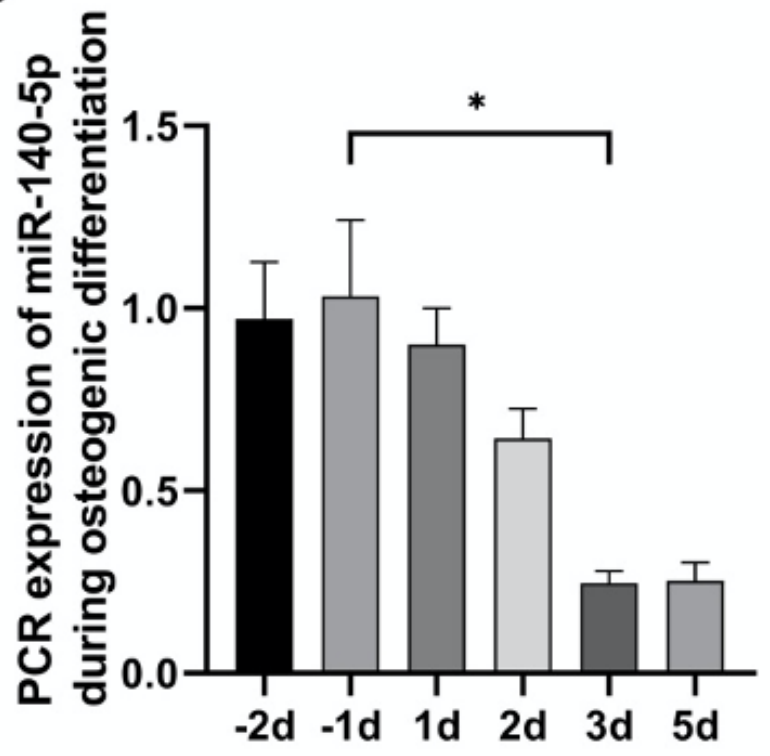

d

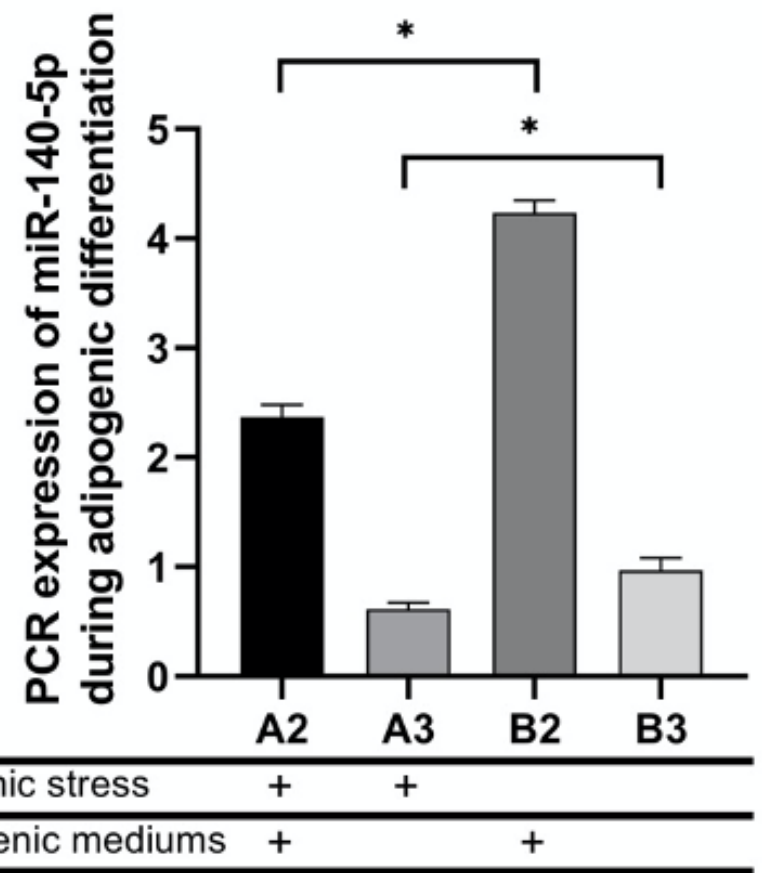

Figure 2

Expression of miR-140-5p in BMSCs is increased by adipogenic induction medium and mechanical stress (stretch stress) and reduced by osteogenic differentiation medium and stretch stress. A) Expression of miR-140-5p during osteogenic differentiation of BMSCs. B) Expression of miR-140-5p during adipogenic differentiation of BMSCs. C) Effects of mechanical stress on miR-140-5p expression in BMSCs during osteogenic differentiation and under non-induction culture conditions. D) Effects of mechanical stress on 
miR-140-5p expression in BMSCs during adipogenic differentiation and under non-induction culture conditions.
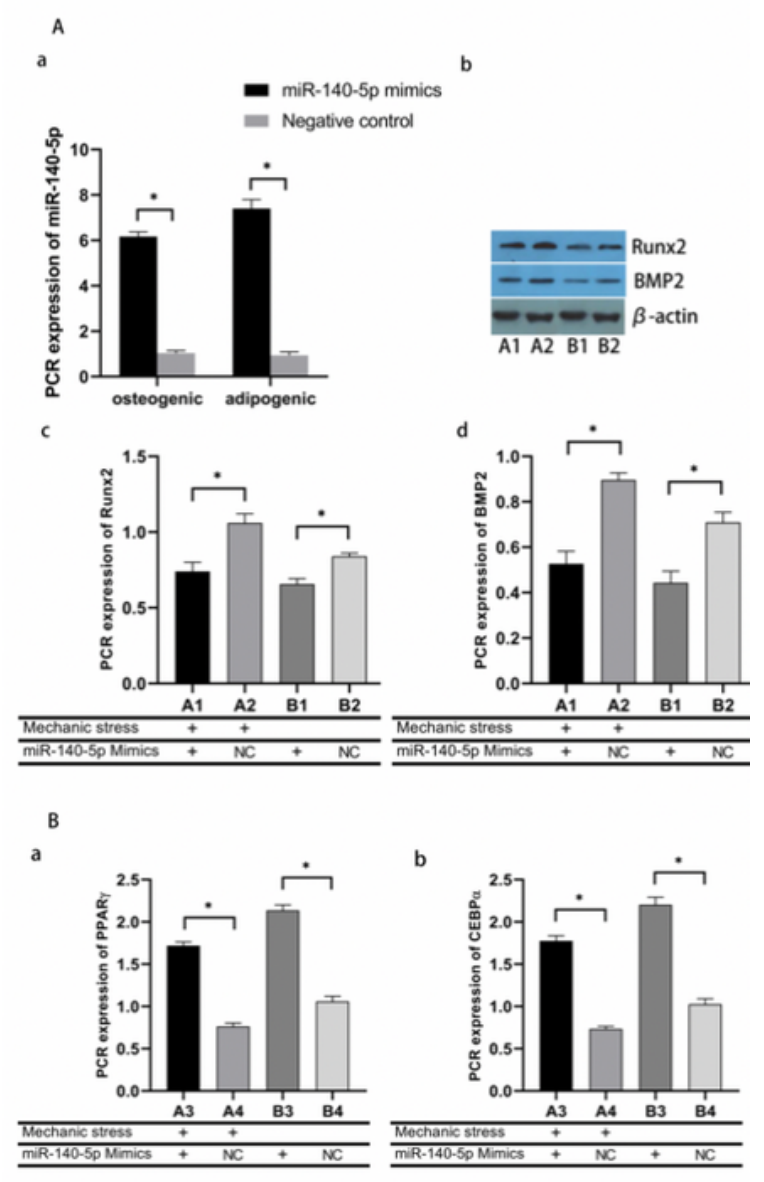

c

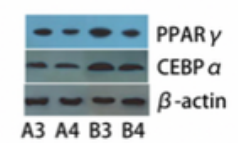

c
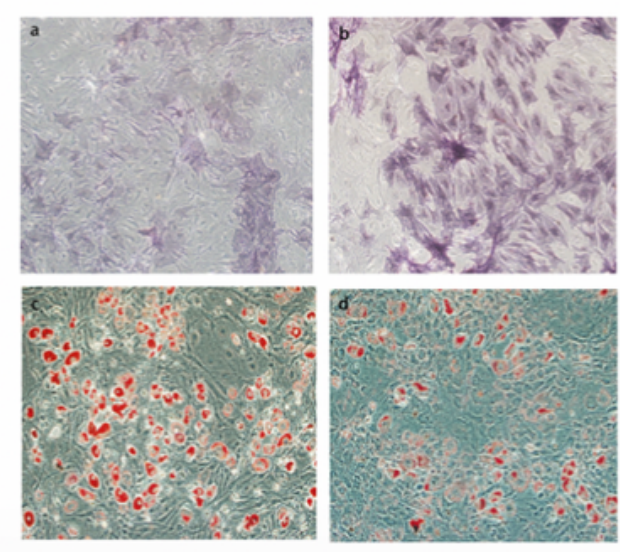

Figure 3

Overexpression of miR-140-5p promotes adipogenic and suppresses osteogenic differentiation of BMSCs. A-a) RT-PCR validation of miR-140-5p mimic overexpression on day 3 of osteogenic and adipogenic culture. A-bcd) RT-PCR and western blot (WB) analyses of osteogenic Runx2 and BMP2 
expression in BMSCs overexpressing miR-140-5p mimic (day 3). B-abc) Expression of adipogenic PPARy and C/EBPa in BMSCs overexpressing miR-140-5p mimic (day 3). C) Alkaline phosphatase (ALP) staining of BMSCs on day 7 of osteogenic culture and oil red 0 staining on day 10 of adipogenic culture.
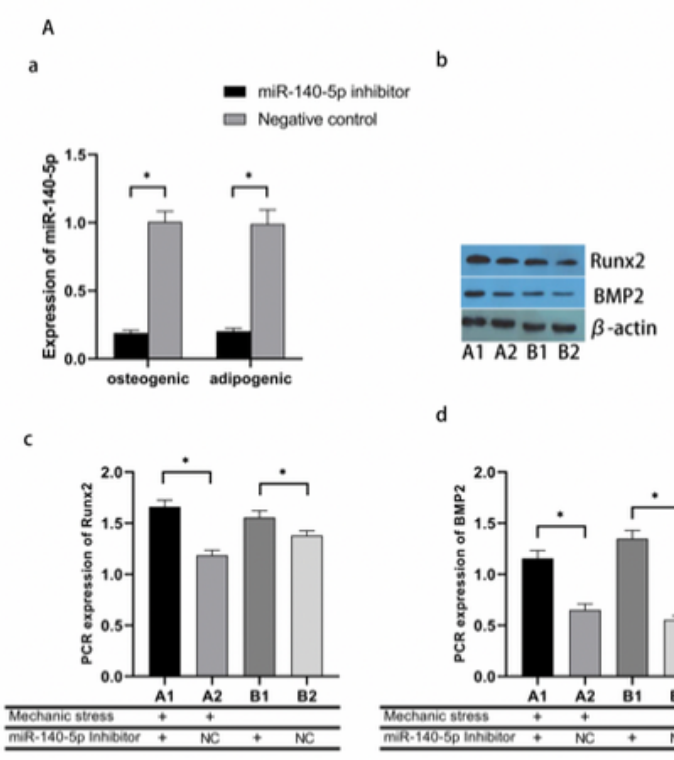

d
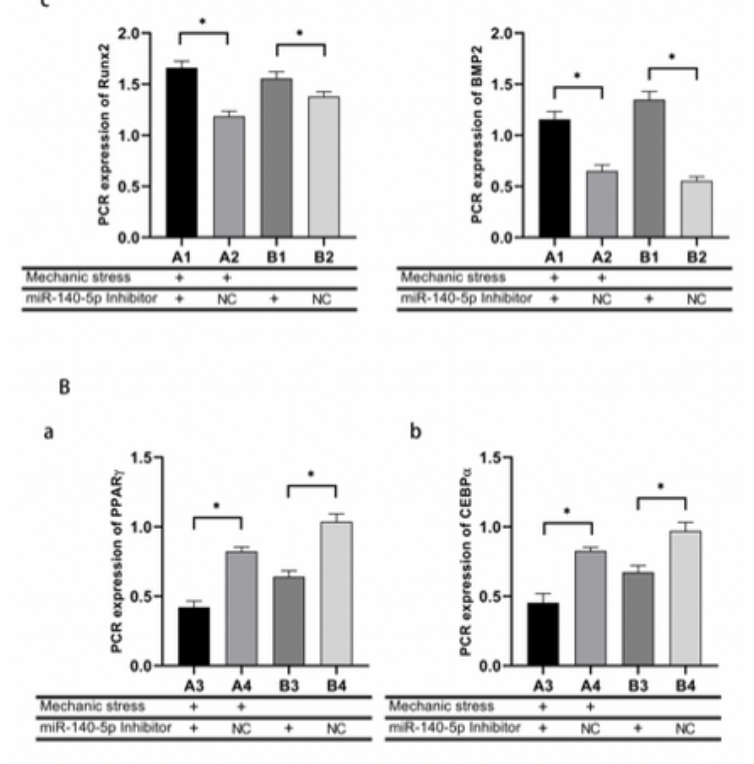

c
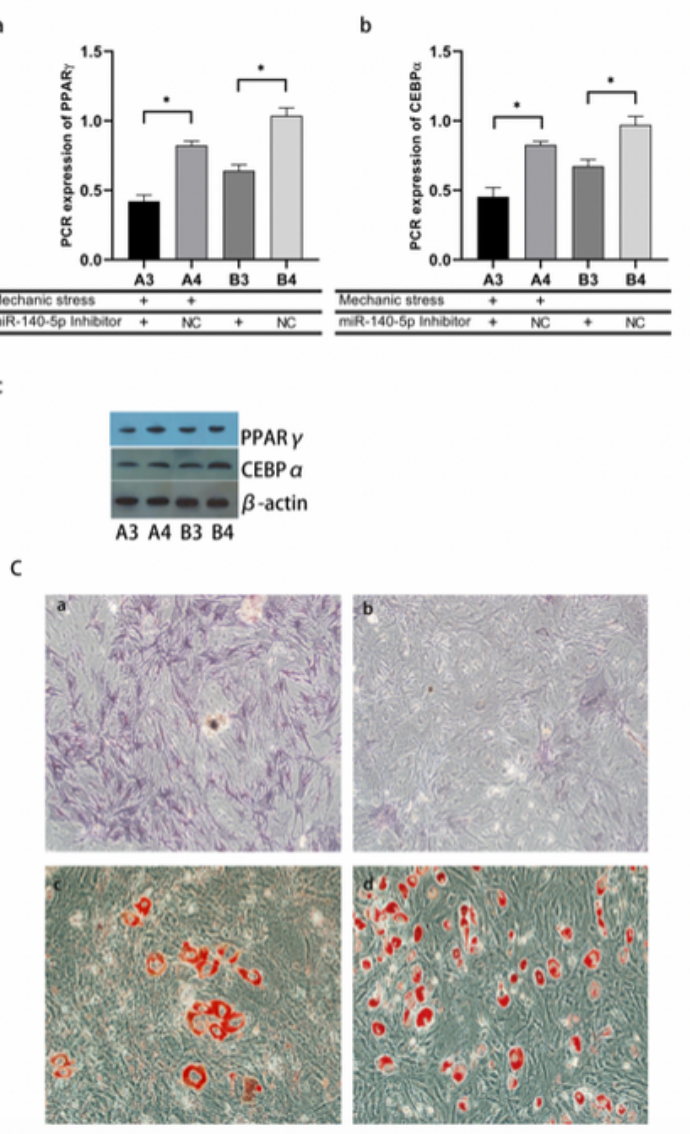

Figure 4

A miR-140-5p inhibitor promotes osteogenic and suppresses adipogenic differentiation of BMSCs. A-a) RT-PCR validation of miR-140-5p knockdown on day 3 under both osteogenic and adipogenic culture 
conditions. A-bcd) RT-PCR and WB analyses of osteogenic Runx2 and BMP2 expression levels in miR140-5P knockdown BMSCs on day 3 compared to cells expressing negative control vector. B-abc) Expression of adipogenic PPARy and C/EBPa in miR-140-5p knockdown BMSCs on day 3. C) ALP staining of BMSCs on day 7 and oil red $O$ staining on day 10 .

a

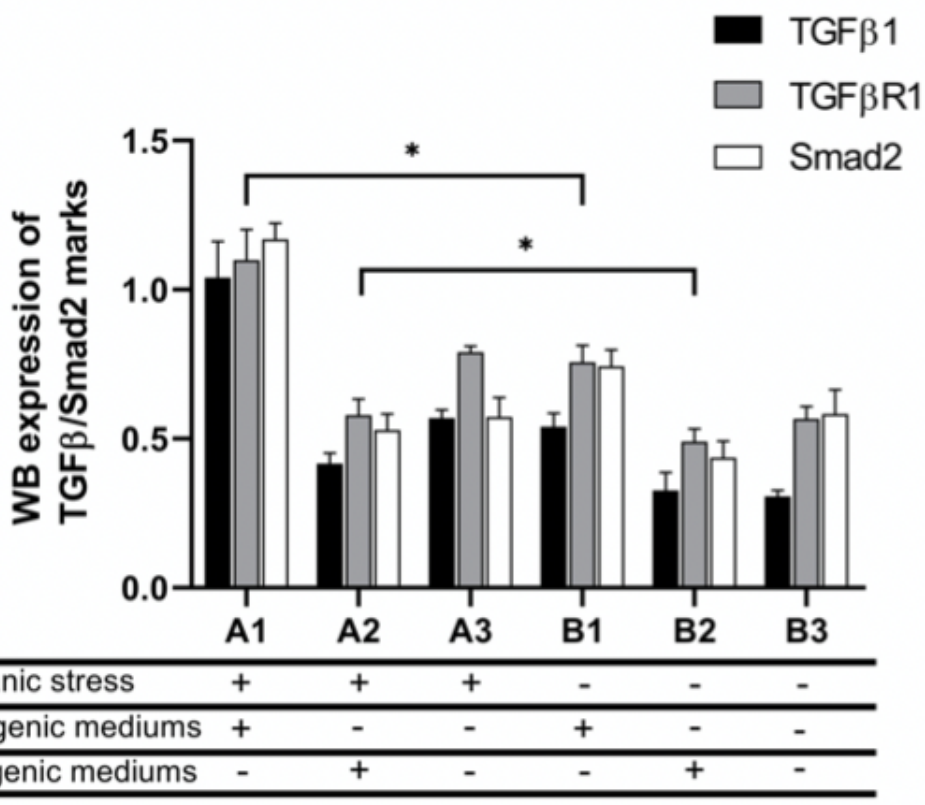

b

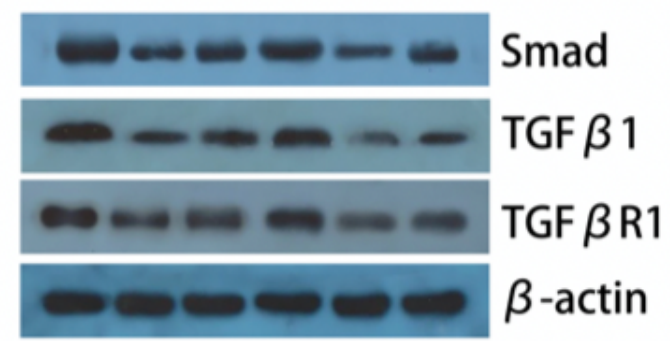

A1 A2 A3 B1 B2 B3

\section{Figure 5}

Stretch stress enhances expression levels of TGFB1/Smad2 signaling pathway components TFG 1 , TFG R1, and Smad2 in BMSCs on day 3. a) WB results for protein expression. b) Sample western blot. 


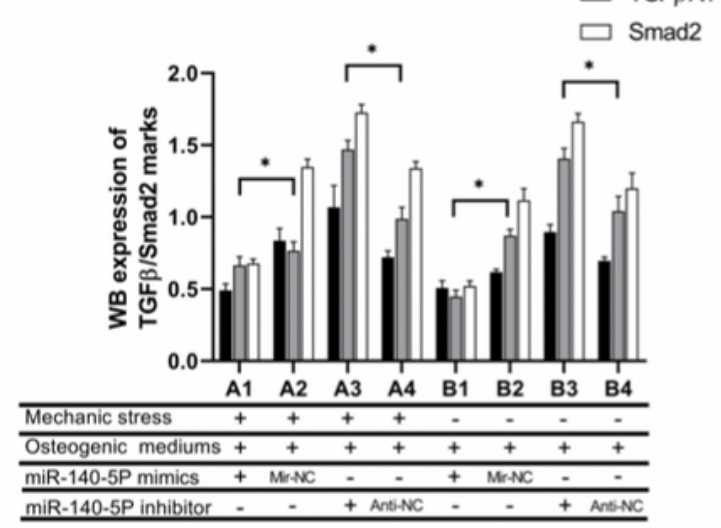

b

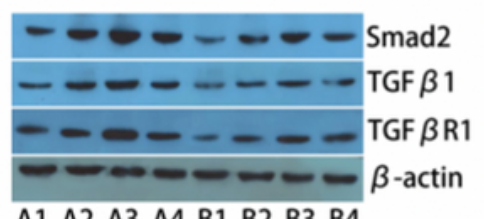

A1 A2 A3 A4 B1 B2 B3 B4

B

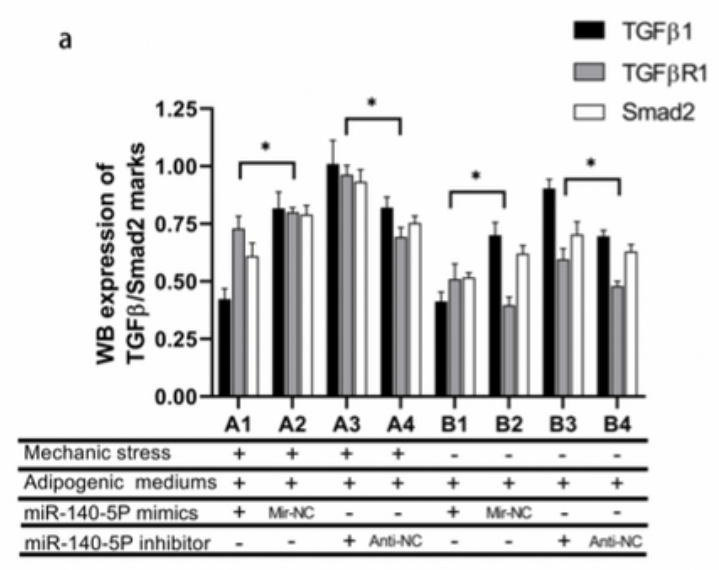

b

$---\infty-\ldots-$ Smad2
$---\ldots-\ldots$ TGF $\beta 1$
$--\infty-\ldots$ TGF $\beta$ R1
$--\infty$-actin
A1 A2 A3 A4 B1 B2 B3 B4

\section{Figure 6}

Stretch stress and miR-140-5p knockdown enhance expression of TGF-1/Smad2 signaling pathway components under osteogenic and adipogenic differentiation conditions. Western blot (WB) results of TGF-1/Smad2 pathway component expression in BMSCs with miR-140-5p overexpression/ inhibition under osteogenic (A) and adipogenic differentiation conditions (B). 


\section{Supplementary Files}

This is a list of supplementary files associated with this preprint. Click to download.

- NC3RsARRIVEGuidelinesChecklistfillable.pdf 